\title{
Electronic structure of amorphous copper iodide: A $p$-type transparent semiconductor
}

\author{
Zhaofu Zhang $\odot,{ }^{1}$ Yuzheng Guo, ${ }^{2}$ and John Robertson ${ }^{1,2, *}$ \\ ${ }^{1}$ Department of Engineering, University of Cambridge, Cambridge, England CB2 1PZ, United Kingdom \\ ${ }^{2}$ School of Electrical Engineering and Automation, Wuhan University, Wuhan 430072, China
}

(Received 26 February 2020; revised manuscript received 10 April 2020; accepted 30 April 2020; published 26 May 2020)

\begin{abstract}
The atomic and electronic structure of the p-type transparent amorphous semiconductor $\mathrm{CuI}$ is calculated by $a b$ initio molecular dynamics. It is found to consist of a random tetrahedrally bonded network. The hole effective mass is found to be quite low, as in the crystal. The valence-band maximum (VBM) state has a mixed $\mathrm{I}(p)-\mathrm{Cu}\left(t_{2 g}\right)-\mathrm{I}(p)$ character, and its energy is relatively insensitive to disorder. An iodine excess creates holes that move the Fermi level into the valence band, but it does not pin the Fermi level above the VBM mobility edge. Thus the Fermi level can easily enter the valence band if $p$ doped, similar to the behavior of electrons in In-Ga-Zn oxide semiconductors but opposite to that of electrons in a-Si:H. This suggests that amorphous $\mathrm{CuI}$ could make an effective $p$-type transparent semiconductor.
\end{abstract}

DOI: 10.1103/PhysRevMaterials.4.054603

\section{INTRODUCTION}

Amorphous oxide semiconductors such as In-Ga-Zn oxide (IGZO) are replacing hydrogenated amorphous silicon $(\mathrm{a}-\mathrm{Si}: \mathrm{H})$ as the main large-area semiconductors for display applications because of their higher electron mobility [1,2]. In a similar fashion, indium tin oxide and polycrystalline $\mathrm{ZnO}$ are heavily used as transparent electrodes in solar cells and displays [3]. However, a weakness of this oxide electronics is that they are all $n$ type, whereas the $p$-type equivalents have much worse performance. $P$-type oxides must counter two problems: they have a large hole effective mass $m^{*}[4,5]$, and they cannot easily be doped because of defect compensation [6,7]. $P$-type oxides of lower $m^{*}$ such as the defossalites like $\mathrm{CuAlO}_{2}$ or related oxides $\mathrm{SrCu}_{2} \mathrm{O}_{2}$ can be designed using the principles of Kawazoe et al. [4], to have a relatively shallow $\mathrm{Cu} d$ core level that interacts with the oxygen $p$-like valence-band maximum (VBM) states to lower their $m^{*}$ value [8-11]. However, the fundamental problem of doping in these oxides is that their VBMs are just too deep below the vacuum level so their acceptor states can be easily compensated [7].

There have been two other approaches to overcome the design of $p$-type oxide semiconductors, first to raise the VBM by using layered oxychalcogenides such as $\mathrm{LaCuOS}$ [12], or by using oxides of lower valence like $\mathrm{SnO}$ or $\mathrm{Cu}_{2} \mathrm{O}$ with occupied $s$-like lone pairs [13-15]. The problem with $\mathrm{SnO}$ and $\mathrm{Cu}_{2} \mathrm{O}$ is that the $\mathrm{Sn}$ and $\mathrm{Cu}$ have a reduced valence so that they have a limited range of stability. At the moment, the $p$-type leg of a halide perovskite is perhaps the simplest $p$-type transparent conductor.

Finally, for large-area electronics such as displays, there is a desire for an amorphous p-type semiconductor for manufacturability, to avoid the problems of grain boundaries. The defossalites have an open layerlike lattice which is

\footnotetext{
*jr@eng.cam.ac.uk
}

unsuitable for amorphization. The LaCuOS-type compounds, being layered, would lose their useful properties if they lost their layers due to disordering. There are a few oxides which can be made amorphous such as $\mathrm{ZnRhO}_{x}$ [16] but these have only moderate mobility but high cost.

There has recently been interest in zinc-blende (zb)-CuI, a $p$-type wide gap compound [17-20]. CuI has a low hole mass and reasonable hole mobility of $8 \mathrm{~cm}^{2} / \mathrm{V} \mathrm{s}$. Interestingly, $\mathrm{CuI}$ with $\mathrm{Sn}$ as an amorphizing agent was recently shown by Jun et al. [21] to be an effective amorphous (a-) semiconductor with similar hole mobility to its crystalline state, and an ability to become a degenerate semiconductor by doping. Thus, a$\mathrm{CuI}$ has the potential to act as a useful $p$-type semiconductor. However, $\mathrm{CuI}$ is a superionic conductor at higher temperatures, which might complicate matters in future.

Here we study the random network models of a-CuI, calculate their electronic structures, and explain how this $p$-type conductor can retain its mobility in the amorphous state, which was previously considered to be achievable only for $s$-like semiconductors [1,22]. Finally, we study why the network does not undergo any reconstructions which constrain its Fermi energy $\left(E_{F}\right)$ to lie within its mobility gap, as would occur by the "Street reconstruction" [23] in amorphous silicon.

\section{METHODS}

The structure of a-CuI is found by using $a b$ initio molecular dynamics (MD) on a supercell of $\mathrm{zb}-\mathrm{CuI}$. We use the VASP and CASTEP plane-wave pseudopotential codes [24,25], with a plane-wave cutoff energy of $700 \mathrm{eV}$, and initially the generalized gradient approximation (GGA) as the exchangecorrelation functional for electrons.

The MD process starts with 44-100 units of $\mathrm{CuI}$, anneals the network at $2000 \mathrm{~K}$ for $4 \mathrm{ps}$, then quenches it at $10 \mathrm{~K} / \mathrm{ps}$ to $300 \mathrm{~K}$. The mass density of a-CuI is taken as $5.40 \mathrm{~g} / \mathrm{cm}^{3}$, $3 \%$ less than for the crystalline phase $\left(5.57 \mathrm{~g} / \mathrm{cm}^{3}\right)$. CuI 
has a relatively low melting point of $606^{\circ} \mathrm{C}$, so the anneal creates a liquidlike structure at $2000 \mathrm{~K}$. The quenching rate is very rapid, and will amorphize the structure, as it does experimentally for glassy metals.

In order to minimize the creation of anomalous features such as gap states or $\mathrm{Cu}$ clustering, it is necessary to keep a large band gap during the MD amorphization process. An alternative $\mathrm{Cu}$ pseudopotential could be generated using the Opium method [26] as in Ref. [11], to create a larger band gap. Alternatively, to partly correct the underestimation of the band gap in density functional theory, here the MD is carried out using a GGA $+U$ potential, with an on-site potential of $U=4.8 \mathrm{eV}$ applied to the closed-shell $\mathrm{Cu} 3 d$ states [27,28]. This lowers the $\mathrm{Cu} 3 d$ states, which then repel the upper valence I $p$ states less, thus widening the band gap. This method is used during the MD, without greatly increasing its computational cost above GGA. This process was used previously for creating a network of a-ZnO [29]. Benavid and Carter [28] find that too large a $U$ value will destabilize the calculation for the case of $\mathrm{Cu}_{2} \mathrm{O}$.

Following $\mathrm{MD}$, the amorphous $\mathrm{CuI}$ structure is relaxed under GGA $+U$ to converge the force on atoms to less than $0.02 \mathrm{eV} / \AA$ and the energy to less than $10^{-5} \mathrm{eV} /$ atom. The electronic partial density of states (PDOS) is then found using the more expensive screened exchange (SX) or HeydScuseria-Ernerzhof (HSE) hybrid functional [30,31]. SX or HSE mixes in a fraction of nonlocal Hartree-Fock exchange into the GGA and they more accurately correct the band-gap error than simple GGA $+U$. An Hartree-Fock fraction of $\alpha=0.33$ is found to raise the HSE06 gap of zb-CuI to the experimental value of $3.1 \mathrm{eV}$ for the VASP potential.

\section{RESULTS}

We first consider the band structure of crystalline zb-CuI. Figure 1(a) shows the band structures of zb-CuI for a VASP potential within the GGA approximation; it has a band gap of only $1.18 \mathrm{eV}$. This is much less than $3.1 \mathrm{eV}$ of the experiment, but typical for many semiconductors with GGA [19]. Figure 1(b) shows the bands for GGA $+U$ with $U=4.8 \mathrm{eV}$, giving a band gap of $1.86 \mathrm{eV}$. We note that the inclusion of $U$ has widened the gap perturbatively by moving down the $\mathrm{Cu} 3 d \Gamma_{12}$ state, which lies at $-2.0 \mathrm{eV}$ below the VBM in the GGA functional [Fig. 1(a)]. This GGA $+U$ gap value is wide enough to be useful for the MD calculation. Figure 1(c) shows the bands of $\mathrm{CuI}$ given by the HSE hybrid functional [31] with a Hartree-Fock exchange content increased from the default $\alpha=25 \%$ to $\alpha=33 \%$, giving a band gap of $3.05 \mathrm{eV}$. Figure 1(d) shows the bands using CASTEP with the SX functional [30], giving a band gap of $3.20 \mathrm{eV}$. Both hybrid functionals show the correct band gap close to the experimental value.

It has been noted that $\mathrm{Cu}$ halides are difficult materials for GW calculations [32,33]. GW based on GGA bands gives a gap of only $2.38 \mathrm{eV}$ for $\mathrm{CuBr}$, whereas $\mathrm{GW}$ of $\mathrm{CuBr}$ based on GGA $+U$ gives a gap of nearer $3.07 \mathrm{eV}$, close to the experimental value. CuI has similar properties. The calculated band-edge line-up to the vacuum level by different functionals is compared in Fig. 1(e). The ionization potential
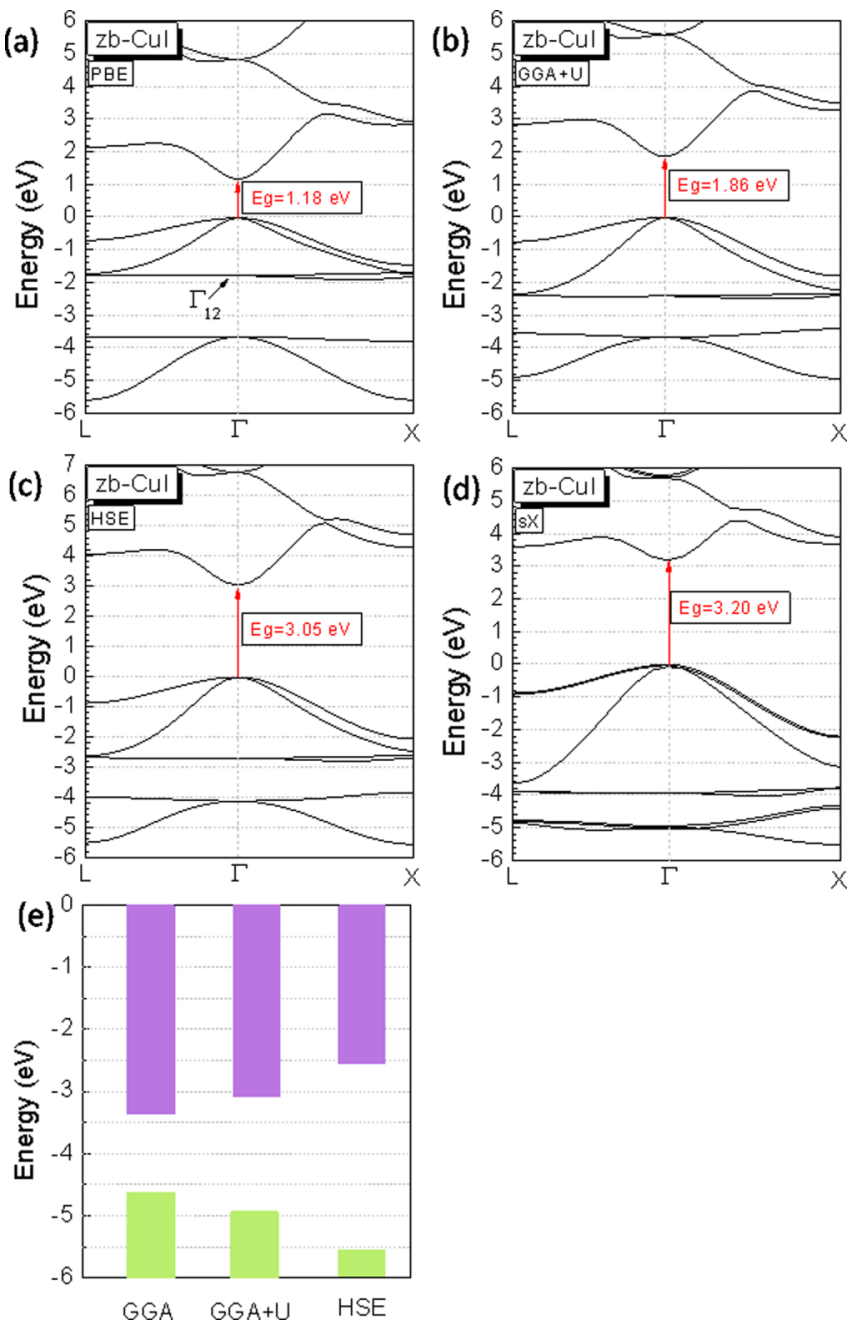

FIG. 1. Band structures of zinc-blende CuI, calculated by (a) PBE, (b) GGA $+U$ with $U=4.8 \mathrm{eV}$, (c) HSE hybrid functional with $33 \%$ Hartree-Fock nonlocal exchange, and (d) SX hybrid functional. (e) Band-edge line-up of zb-CuI by different functionals, where the vacuum level is at $0 \mathrm{eV}$.

was calculated by forming a zb-CuI slab with nonpolar (110) faces. CuI atoms have a Bader charge of \pm 0.28 .

Figure 2(a) shows the continuous random network model of a-CuI derived from a VASP MD calculation with GGA + $U(U=4.8 \mathrm{eV})$ and subsequent relaxation also with $U=$ $4.8 \mathrm{eV}$. The $\mathrm{Cu}$ atoms are copper colored, iodide atoms are purple, and any Sn atoms used in later models are shown as blue. zb-CuI has tetrahedral $\mathrm{Cu}$ and I sites, with a $\mathrm{Cu}-\mathrm{I}$ bond length of $2.64 \AA$. In the a-CuI structure of Fig. 2, the mean $\mathrm{Cu}-\mathrm{I}$ bond length is $2.7 \AA$.

The network of a-CuI has a tetrahedral structure with few like-atom bonds. Figure 3(a) shows the partial radial distribution functions (RDFs) of this network. In addition to the first-neighbor $\mathrm{Cu}-\mathrm{I}$ peak at $2.70 \AA$, there is a smaller $\mathrm{Cu}-\mathrm{Cu}$ second-neighbor peak at $2.8 \AA$, which also extends to larger distances. The analogous I-I distribution starts only at $3.7 \AA$, indicating the absence of direct I-I bonds in this network. The network size is relatively small for cost reasons because we are primarily interested in obtaining the first-neighbor 


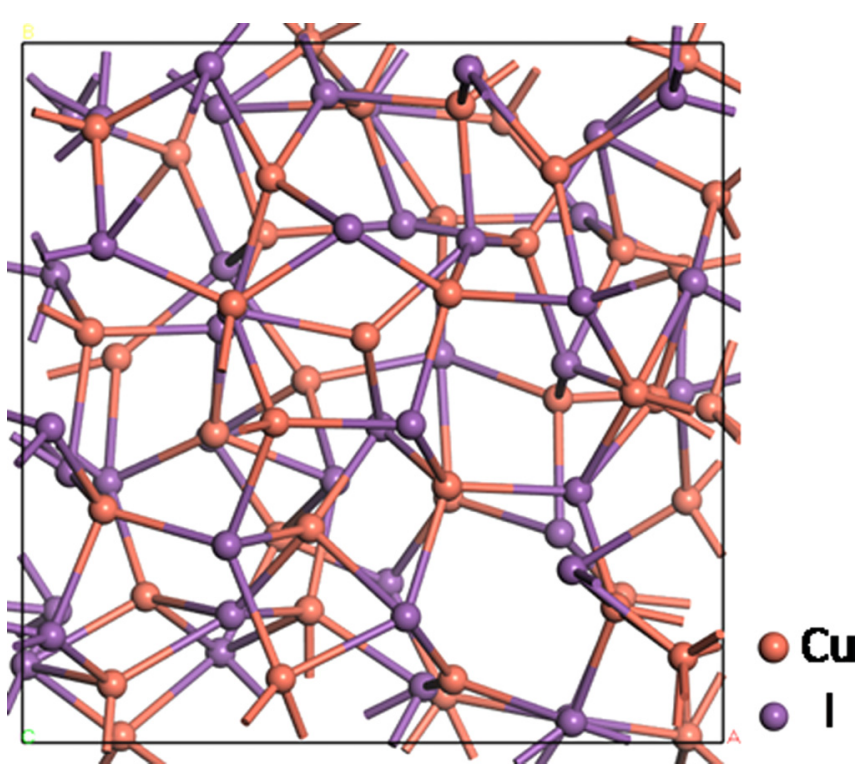

FIG. 2. Random network of $\mathrm{a}-\mathrm{Cu}_{44} \mathrm{I}_{44}$ created by molecular dynamics with $\mathrm{GGA}+U(U=4.8 \mathrm{eV})$ in $\mathrm{MD}$ and relaxation processes.

coordination, either tetrahedral or trigonal, and the degree of $\mathrm{Cu}$ clustering. The intrinsic width of the first-neighbor peak of a-CuI is larger than typical amorphous solids, due to the lower rigidity of $\mathrm{CuI}\left(c_{11}=45.1 \mathrm{GPa}\right)$ [17] compared to $c_{11}=165.6 \mathrm{GPa}$ of Si [34].

We have calculated a-CuI networks by the MD procedure from 44 to $100 \mathrm{CuI}$ formula units. The latter calculations are quite time consuming. The basic features are not found to change, including the width of the first-neighbor peak. It is recognized that the smaller networks are too small for accurate RDFs, and larger ones will be developed in due course.

The main defects in this network are threefold coordinated sites, as was the case in a-GaAs [35,36], a-GaN [37], and $\mathrm{a}-\mathrm{ZnO}$ [29]. The calculated local structure is sensitive to the density taken for a-CuI. Lower values can cause the a-CuI network to develop a locally layerlike structure with trivalent sites. These arise from bonding resembling $\mathrm{CuI}$ polytypes such as the rhombohedral $(\mathrm{R} \overline{3} \mathrm{c})$ and "antilitharge" $(P 4 / \mathrm{nmm})$ tetragonal structure $[38,39]$.

An unusual feature of a-CuI discussed later, shown in Fig. S2 in the Supplemental Material [38], is the presence of internal $\mathrm{Cu}-\mathrm{Cu}$ bonds and $\mathrm{Cu}$ clusters in addition to the usual $\mathrm{Cu}-\mathrm{I}$ bonds. These arise because $\mathrm{CuI}$ is a superionic conductor in its higher-temperature phases. The $\mathrm{Cu}$ ion is mobile amongst a relatively stationary framework of larger iodide ions [40-42]. The incipient motion of $\mathrm{Cu}$ ions leads to this $\mathrm{Cu}-\mathrm{Cu}$ bonding and clustering, but this aspect is inconvenient to its role as a semiconductor.

The $\mathrm{Cu}-\mathrm{Cu}$ bonds can become a significant feature in the MD when carried out with potentials giving a small band gap like $1.2 \mathrm{eV}$, but they disappear if the band gap during MD exceeds $\approx 1.8 \mathrm{eV}$. The clusters occur for smaller gap values, as hole polarons form with the Fermi level dropping into the valence band. The atomic positions of $\mathrm{Cu}$ atoms of a reasonable a-CuI model are highlighted in Fig. 3(b), where the $\mathrm{I}$ atoms are hidden, and the $\mathrm{Cu}-\mathrm{Cu}$ bonds with a length cutoff

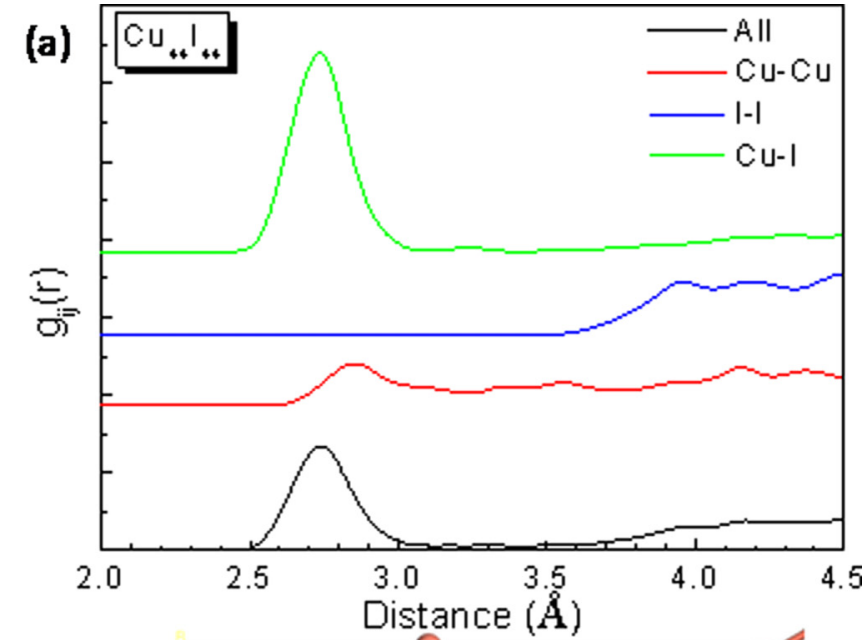

(b)

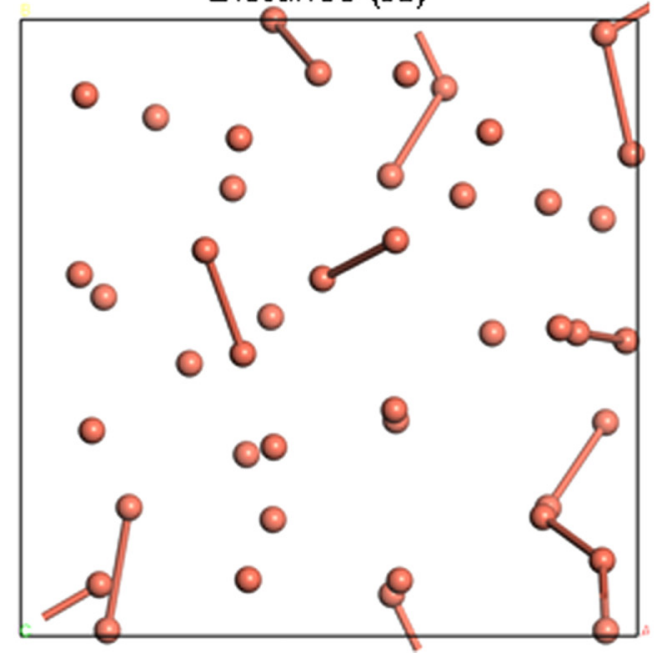

FIG. 3. Partial radial distribution functions of the a-Cu $\mathrm{Cu}_{44} \mathrm{I}_{44}$ network of Fig. 2. (b) Network of Fig. 2 showing the $\mathrm{Cu}-\mathrm{Cu}$ connections with cutoff length of $3.0 \AA$, indicating the negligible presence of $\mathrm{Cu}-\mathrm{Cu}$ clusters.

of 3.0 $\AA$ are connected (longer than the averaged $\mathrm{Cu}-\mathrm{Cu}$ bond length of $2.8 \AA$ ) in Fig. 3(a), showing the low evidence of direct $\mathrm{Cu}-\mathrm{Cu}$ bonding in this network.

Figure 4(a) shows the calculated GGA $+U$ density of states for this network. The Fermi level lies in the midgap, so it is a semiconductor with a clean gap of $2.0 \mathrm{eV}$. Figure 4(b) shows the calculated PDOS of a-CuI by the HSE functional. This PDOS is overall quite similar to the PDOS of crystalline CuI, with a band gap of $3.0 \mathrm{eV}$, shown in Fig. 4(c). The main $\mathrm{Cu} d$ peak of nondispersive $\Gamma_{12}$ states lies at $-2.9 \mathrm{eV}$ in the valence band, in between two peaks due to interacting $\mathrm{I} p-\mathrm{Cu}$ $d$ states. The lowest conduction band (CB) is mainly $\mathrm{Cu} s$ states. The higher $\mathrm{CB}$ consists of $\mathrm{Cu} p$ states. An effective hole mass can be estimated from the parabolic shape of the VBM DOS using $N(E) \sim\left(m^{*} E\right)^{1 / 2}$, and is about $0.3 m_{0}$, roughly as measured, and similar to the crystalline value. Thus, a-CuI has a relatively small hole $m^{*}$, like the crystalline phase [17], unlike $\mathrm{Cu}$ oxide compounds. The small hole effective mass guarantees the considerable hole mobility, which is significant for electronics applications.

The heat formation of $\mathrm{zb}-\mathrm{CuI}$ is calculated to be $-0.76 \mathrm{eV}$ per $\mathrm{CuI}$ formula unit in the $\mathrm{SX}$ functional, close to a previous 

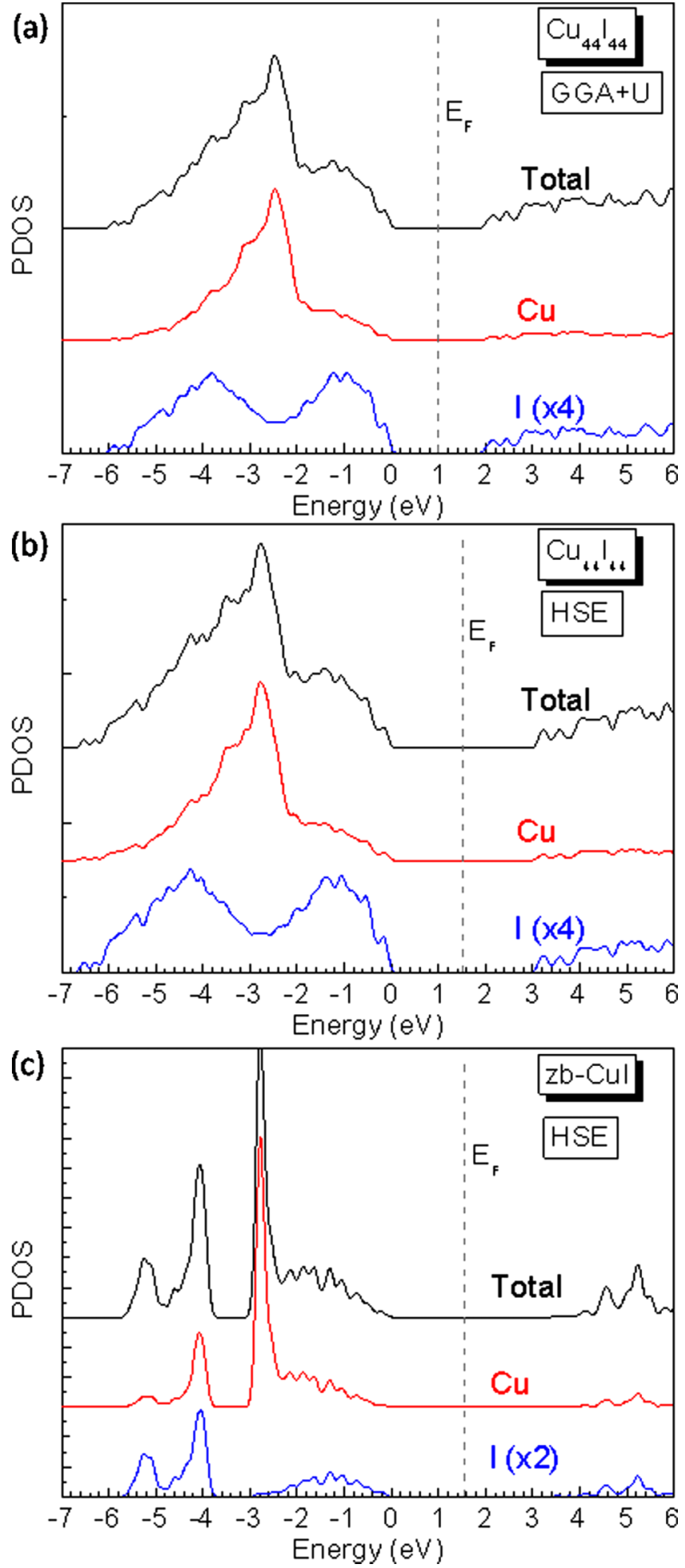

FIG. 4. Partial density of states of the a- $\mathrm{Cu}_{44} \mathrm{I}_{44}$ network of Fig. 2, calculated by (a) GGA $+U$ and (b) HSE hybrid functional. (c) PDOS of zb-CuI on the same scale for comparison. Note $E_{F}$ in the midgap. The PDOS of I is enlarged to make it more obvious.

report [20], and $-0.46 \mathrm{eV}$ per formula unit for the amorphous phase. A similar amorphization energy was found for $\mathrm{a}-\mathrm{ZnO}$ [29].

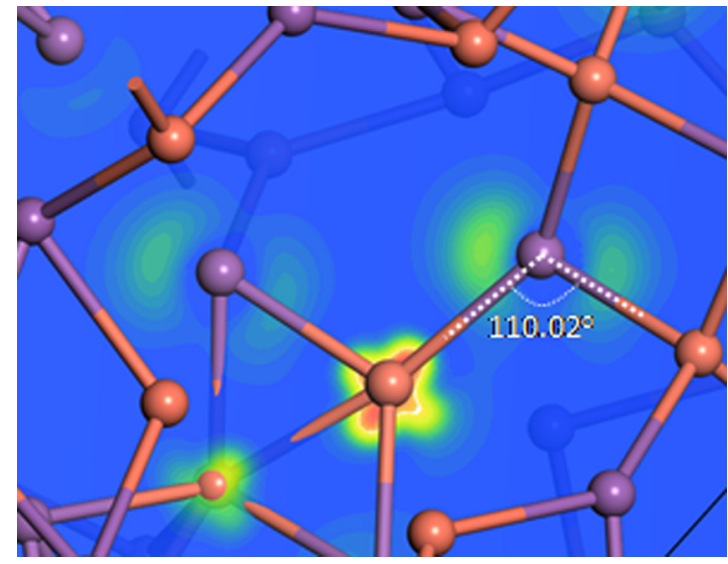

FIG. 5. Wave function of the valence-band maximum state in the a- $\mathrm{Cu}_{44} \mathrm{I}_{44}$ network of Fig. 2.

We now discuss why the VBM of a-CuI is relatively insensitive to disorder, and its hole effective mass is similar to that of the crystal. This aspect resembles how the CBM of a-IGZO is insensitive to disorder. This effect in the metal oxides has been attributed to the cation $s$-like character of its CBM [2,22], but that is only a partial explanation. The CBM states of IGZO and $\mathrm{SnO}_{2}[43,44]$ consist almost entirely of cation $s$ states and almost no anion states. The CBM wave function consists of a symmetric combination of these $s$ states. This causes the phase of each atomic orbital contribution to be positive, irrespective of the ring order, whether it is sixfold (even) or fivefold (odd). On the other hand, a ring of $s p^{3}$ hybrids would have a sign clash around any fivefold ring of bonds if it tries to have any antibonding character, as a conduction-band state would normally have. This is a significant disorder and would create a Hall effect sign anomaly, as in Friedman's model [44]. The sign consistency for all ring orders of $s$ states causes no disorder for the case of a-IGZO.

Turning to a-CuI, Jun et al. [21] suggest that the low sensitivity of the VBM states of a-CuI to disorder is due to its iodide $p$ orbitals at the VBM acting in a quasi-s-like manner. However, this is unlikely. We know that the VBM state of crystalline $\mathrm{CuI}$ has a mixed $\mathrm{I}(p)-\mathrm{Cu}(d)$ character [18], like that of $\mathrm{CuAlO}_{2}$ [11]. It does not originate from just the anion. This is also true of the VBM state in the a-CuI phase, as seen by the wave function in Fig. 5. The a-CuI network consists of two-center bonds of $\mathrm{Cu}$ and $\mathrm{I} s p^{3}$ hybrids, like a-GaAs. Its VBM is three near-degenerate states. If we consider a plane of three atoms centered on a tetrahedral $\mathrm{Cu}$ site, the orbital has local $\mathrm{I}(p)-\mathrm{Cu}\left(t_{2 g}\right)-\mathrm{I}(p)$ character, with the $\mathrm{I}(p)$ states lying parallel, as shown in Fig. 5. Overall, this I-Cu-I unit could be less sensitive to disorder, in that its energy above the nonbonding $\mathrm{Cu} 3 d \Gamma_{12}$ states remains relatively constant, the orbital being more delocalized. This is seen by comparing the calculated PDOS of each phase, using the orbital analysis of Goldmann et al. [18].

We now consider the effect of stoichiometry on the a-CuI network. Amorphous $\mathrm{CuI}$ is an intrinsic semiconductor with $E_{F}$ lying in the midgap (Fig. 4). On the other hand, Fig. 6(a) shows the network of I-rich a-CuI $\mathrm{C}_{1+x}$ with a two-atom iodine excess. The DOS shows that the $E_{F}$ moves into the valence 


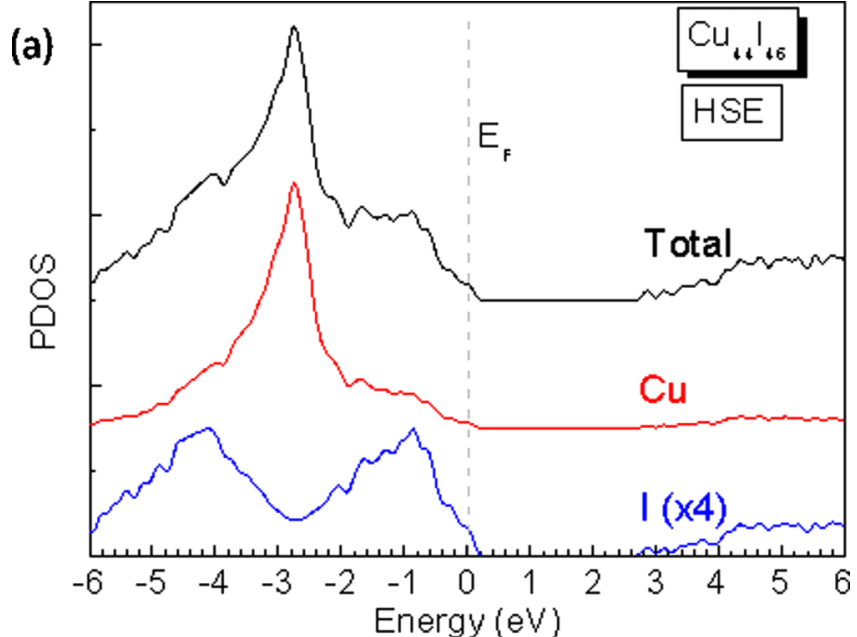

(b)

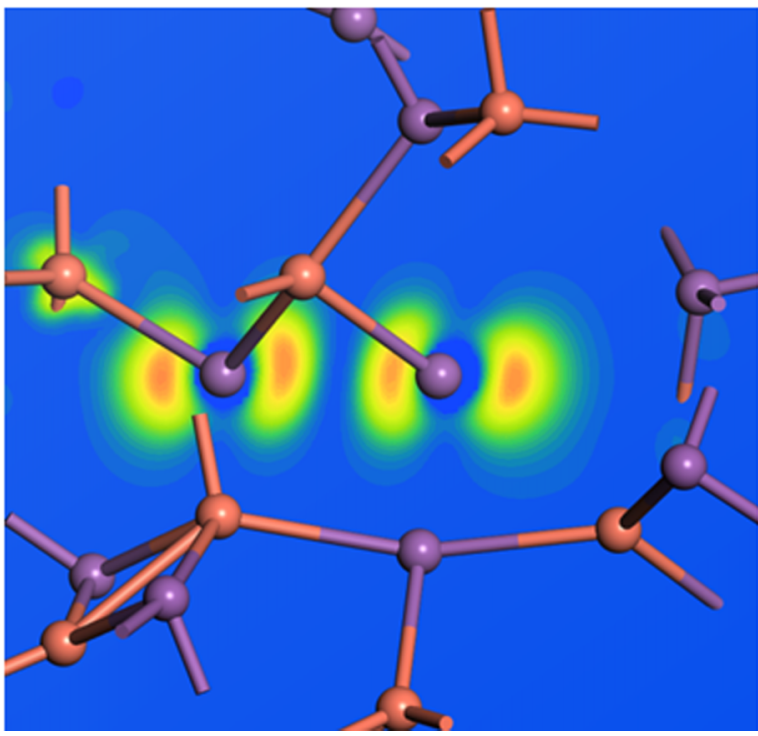

FIG. 6. (a) Partial density of states of the I-excess a-Cu $\mathrm{Cu}_{44} \mathrm{I}_{46}$ network. Note $E_{F}$ lies in the upper valence band. (b) Wave function of the defect state at the top of the valence band in the I-excess network. The wave function is localized on two iodine sites.

band. The upper valence states form a single, continuous band with the main valence band. Notably, they do not create a split-off defect band above the VBM, with $E_{F}$ lying in a "minigap" above the main valence band, thus pinning $E_{F}$ above the valence-band mobility edge. There is some network rearrangement to accommodate the iodine excess for the a$\mathrm{CuI}_{1+x}$, but there is no reconstruction, similar to the Street process [23], to cause Fermi level pinning above the mobility edge. Thus there is no creation of localized trap states.

The wave function of this hole state of $\mathrm{a}-\mathrm{CuI}_{1+x}$ is shown in Fig. 6(b). It is localized mainly on orbitals on two I sites with parallel orientation. Thus, excess holes in $\mathrm{a}-\mathrm{CuI}_{1+x}$ behave like excess electrons in a-IGZO, where experimentally the Hall electron mobility increases continuously with increasing free-electron numbers $[1,45]$. This behavior is in contrast to the behavior of doped a-Si:H. There, the bonding network on $n$-type doped a-Si:H undergoes a reconstruction following the model of Street [23]. This reconstruction pins $E_{F}$ below its conduction-band mobility edge, so $E_{F}$ remains in localized (a)

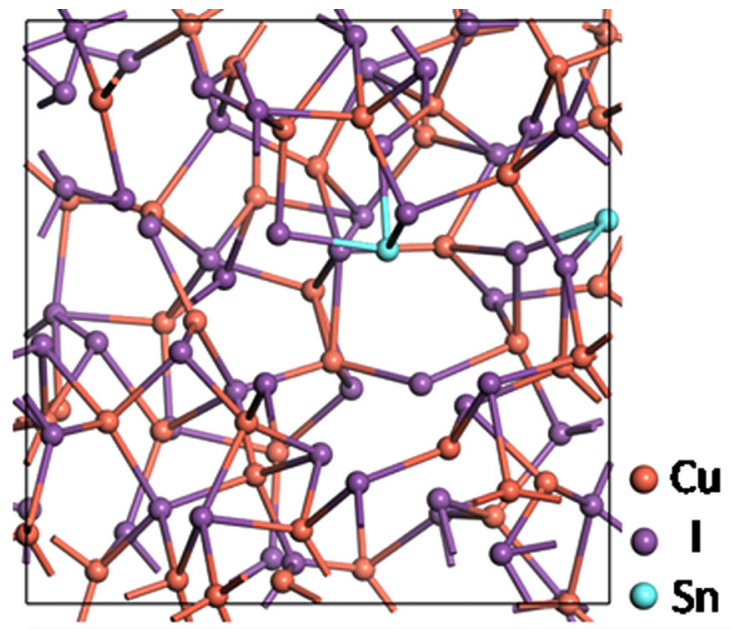

(b)

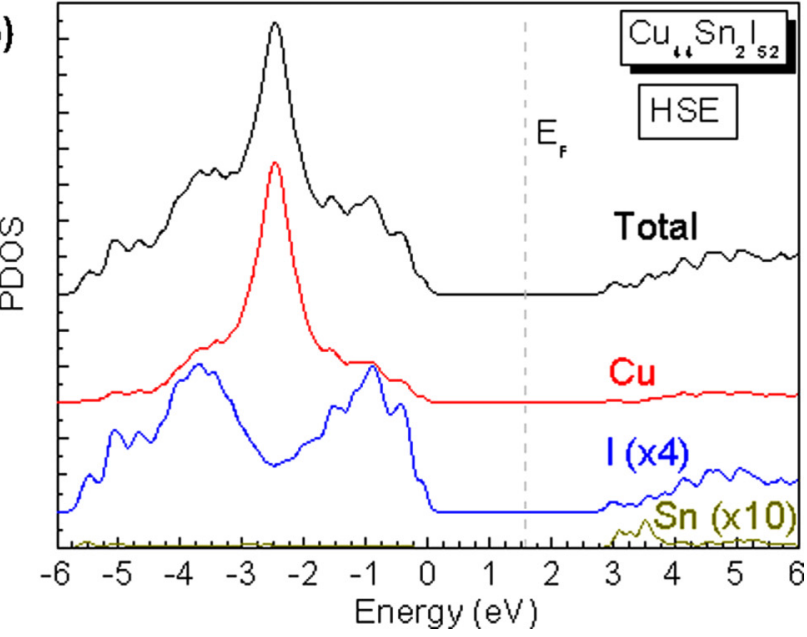

FIG. 7. (a) The network of $\mathrm{Cu}_{44} \mathrm{Sn}_{2} \mathrm{I}_{52}$ with $\mathrm{Sn}$ as amorphizing agent. Note the greater number of threefold I sites, making a more layered structure. (b) PDOS and of this $\mathrm{Cu}_{44} \mathrm{Sn}_{2} \mathrm{I}_{52}$ network. Note $E_{F}$ in the midgap, as in the case of Fig. 4.

states. This is a critical process as it limits the field-effect mobility of a-Si: $\mathrm{H}$ thin-film transistors to small values characteristic of localized states, and it is the key difference between the behavior of a-Si:H and a-IGZO [22]. Amorphous CuI follows the a-IGZO behavior, for its holes, and leads to larger hole mobility values.

A network of a-Cu${ }_{44} \mathrm{Sn}_{2} \mathrm{I}_{52}$ has been simulated by including the Sn amorphizing agent used experimentally by Jun et al. [21], as shown in Fig. 7(a). Sn atoms are added to the $\mathrm{CuI}$ supercell with its size adjusted to maintain the density. $\mathrm{Sn}$ is not needed to amorphize $\mathrm{CuI}$ in the simulation of pure a-CuI because of the high quenching rate in the MD process. Experimentally, Sn was in the +4 charge state according to $\mathrm{x}$-ray photoemission spectroscopy, so the $\mathrm{SnI}_{4}$ unit satisfies the stoichiometry. The calculations find that $\mathrm{Sn}$ does not affect the local bonding arrangements of $\mathrm{Cu}$ and I much; the calculated density of states are similar to that without $\mathrm{Sn}$ doping, as shown in Fig. 7(b). As can be seen in the PDOS in Fig. 7(b), the $E_{F}$ lies in the midgap, as in the case of a- $\mathrm{Cu}_{44} \mathrm{I}_{44}$ in Fig. 4(b). This is due to the ideal stoichiometry by the $\mathrm{SnI}_{4}$ unit. The VBM wave function of a-CuSnI is similar to 
that in Fig. 5, with localized states of local $\mathrm{I}(p)-\mathrm{Cu}\left(t_{2 g}\right)-\mathrm{I}(p)$ character and parallel-orientated I atoms.

The presence of $\mathrm{Cu}-\mathrm{Cu}$ bonds and the appearance of internal $\mathrm{Cu}$ clusters are features of $\mathrm{CuI}$ related to its hightemperature superionic behavior [39-41]. The $\mathrm{Cu}$ ions are mobile within a framework of the larger iodide ions. There has been great discussion over the migration path of the $\mathrm{Cu}$ ions. $\mathrm{Cu}$ clustering is a precursor of the $\mathrm{Cu}$ migration path.

$\mathrm{The} \mathrm{Cu}-\mathrm{Cu}$ distribution in our a-CuI networks can show the formation of $\mathrm{Cu}-\mathrm{Cu}$ bonds. These can be of variable length, some as short as the primary $\mathrm{Cu}-\mathrm{I}$ bonds. The $\mathrm{Cu}-\mathrm{Cu}$ bonds can create $\mathrm{Cu}$ clusters within the main $\mathrm{Cu}-\mathrm{I}$ network. These bonds have the effect of broadening the $\mathrm{Cu} 3 d$ band. As a result, larger clusters can cause $E_{F}$ to enter the upper valence band, thus forming a different random network.

Direct $\mathrm{Cu}-\mathrm{Cu}$ bonds are generally an error of the $\mathrm{MD}$ process due to the underestimation of the GGA band gap of $\mathrm{CuI}$, which allows holes to form too easily in the a-CuI VBM. Cu potentials often give a GGA gap of only $\approx 1.1 \mathrm{eV}$ for zb-CuI [19]. An MD calculation with such narrow gap will allow $\mathrm{Cu}-\mathrm{Cu}$ bonds to form, which are not found when other potentials are used, such as those created by the Opium method [26,11]. Alternatively, including $U=4.8 \mathrm{eV}$ in the calculation increases the GGA band gap of zb-CuI to $1.86 \mathrm{eV}$, as shown in Fig. 1(b). This wider gap can inhibit the cluster formation in $\mathrm{MD}$, so the $\mathrm{Cu}-\mathrm{Cu}$ interactions do not form such short bonds [Fig. 3(b)].

An example of the a- $\mathrm{Cu}_{44} \mathrm{I}_{44}$ network with $U=0 \mathrm{eV}$ in the MD process is shown in Fig. S1(a) in Ref. [38]. A large $\mathrm{Cu}$ cluster is formed within this network [Fig. S1(b) in Ref. [38]] because of the severely underestimated gap value by GGA. Bonding analysis from the RDF curve [Fig. S1(c) in Ref. [38]] shows that the average $\mathrm{Cu}-\mathrm{Cu}$ bond length in this case is $2.55 \AA$, much shorter than in Fig. 3(a). These $\mathrm{Cu}$ clusters cause $E_{F}$ to be pinned in the valence band, rather than the correct midgap position, as verified in the PDOS in Fig. S1(d) in Ref. [38].

Figure S2 in Ref. [38] compares the effect of different $U$ values in the MD process on the final a-CuI network, including the $\mathrm{Cu}$ coordination number [Fig. S2(a) in Ref. [38]], the averaged $\mathrm{Cu}-\mathrm{Cu}$ bond length [Fig. S2(b) in Ref. [38]], the $\mathrm{Cu}$ cluster size [Fig. S2(c) in Ref. [38]], and the electronic structures including band gap and $\mathrm{Cu} d$ orbital position [Fig. S2(d) in Ref. [38]]. Clearly, with the increase of $U$ value in the $\mathrm{MD}$ process, the $\mathrm{Cu}-\mathrm{Cu}$ averaged bond length becomes longer, and the $\mathrm{Cu}$ cluster size becomes smaller. Thus $\mathrm{Cu}$ cluster formation can be effectively avoided by maintaining a large $U$ during MD. With the overall consideration, we chose $U=4.8 \mathrm{eV}$ in our paper.

In summary, $a b$ initio molecular dynamics is used to calculate the atomic and electronic structure of amorphous $\mathrm{CuI}$, which was recently shown to be a transparent amorphous p-type semiconductor. The amorphous phase of $\mathrm{CuI}$ is found to have a similar bonding to the crystalline phase. The hole mass of a-CuI is found to be relatively low like its crystalline parent, and insensitive to disorder. The valence-band maximum is found to have mixed orbital character, consisting of $\mathrm{I}(p)-\mathrm{Cu}\left(d_{x y}\right)$ multicenter orbitals. The presence of hole states due to excess I or shifting the Fermi level in a transistor does not cause a reconstruction that pins $E_{F}$ above the VB mobility edge, so that a-CuI is potentially very useful as a $p$-type amorphous transparent semiconductor.

\section{ACKNOWLEDGMENT}

J.R. acknowledges funding from EPSRC Grant No. $\mathrm{EP} / 005152 / 1$.
[1] H. Hosono, M. Yasukawa, and H. Kawazoe, J. Non-Cryst. Solids 203, 334 (1996); H. Hosono, ibid. 352, 851 (2006).

[2] T. Kamiya and H. Hosono, NPG Asia Mater. 2, 15 (2010).

[3] K. Ellmer, Nat. Photonics 6, 809 (2012).

[4] H. Kawazoe, M. Yasukawa, H. Hyodo, M. Kurita, H. Yanagi, and H. Hosono, Nature (London) 389, 939 (1997).

[5] G. Hautier, A. Miglio, G. Ceder, G. M. Rignanese, and X. Gonze, Nature Commun. 4, 2292 (2013).

[6] S. Lany, J. Osorio-Guillen, and A. Zunger, Phys. Rev. B 75, 241203(R) (2007).

[7] J. Robertson and S. J. Clark, Phys. Rev. B 83, 075205 (2011).

[8] A. Kudo, H. Yanagi, H. Hosono, and H. Kawazoe, Appl. Phys. Lett. 73, 220 (1998).

[9] H. Ohta, M. Orita, M. Hirano, I. Yagi, K. Ueda, and H. Hosono, J. Appl. Phys. 91, 3074 (2002).

[10] J. Robertson, P. W. Peacock, M. D. Towler, and R. J. Needs, Thin Solid Films 411, 96 (2002).

[11] R. Gillen and J. Robertson, Phys. Rev. B 84, 035125 (2011).

[12] K. Ueda, S. Inoue, S. Hirose, H. Kawazoe, and H. Hosono, Appl. Phys. Lett. 77, 2701 (2000).

[13] Y. Ogo, H. Hiramatsu, K. Nomura, H. Yanagi, T. Kamiya, M. Hirano, and H. Hosono, Appl. Phys. Lett. 93, 032113 (2008).
[14] E. Fortunato, V. Figueiredo, P. Barquinha, E. Elamurunga, R. Barros, G. Goncalves, S. H. K. Park, C. S. Hwang, and R. Martins, Appl. Phys. Lett. 96, 192102 (2010).

[15] D. O. Scanlon, B. J. Morgan, G. W. Watson, and A. Walsh, Phys. Rev. Lett. 103, 096405 (2009); E. Fortunato, P. Barquinha, and R. Martins, Adv. Mater. 24, 2945 (2012).

[16] S Narushima, K Ueda, H Mizoguchi, H Ohta, M Hirano, K Shimizu, T Kamiya, and Hosono, Adv. Mater. 15, 1409 (2003).

[17] M. Grundmann, F. L. Schein, M. Lorenz, T. Böntgen, J. Lenzner, and H. Wenckstern, Phys. Status Solidi A 210, 1671 (2013).

[18] A. Goldmann, J. Tejeda, N. J. Shevchik, and M. Cardona, Phys. Rev. B 10, 4388 (1974).

[19] J. Wang, J. Li, and S. S. Li, J. Appl. Phys. 110, 054907 (2011).

[20] M. Grauzinyte, S. Botti, M. A. L. Marques, S. Goedecker, and J. A. Flores-Livas, Phys. Chem. Chem. Phys. 21, 18839 (2019).

[21] T. Jun, J. Kim, M. Sasase, and H. Hosono, Adv. Mat. 30, 1706573 (2018).

[22] J. Robertson, Phys. Status Solidi B 245, 1026 (2008).

[23] R. A. Street, Phys. Rev. Lett. 49, 1187 (1982).

[24] G. Kresse and J. Furthmüller, Phys. Rev. B 54, 11169 (1996); Comput. Mater. Sci. 6, 15 (1996). 
[25] S. J. Clark, M. D. Segall, C. J. Pickard, P. J. Hasnip, M. J. Probert, K. Refson, and M. C. Payne, Z. Kristallogr. 220, 567 (2005).

[26] A. M. Rappe, K. M. Rabe, E. Kaxiras, and J. D. Joannopoulos, Phys. Rev B 41, 1227 (1990).

[27] A. Janotti, D. Segev, and C. G. Van de Walle, Phys. Rev. B 74, 045202 (2006).

[28] L. I. Benavid and E. A. Carter, J. Phys. Chem. C 117, 26048 (2013); K. Yu, and E. A. Carter, J. Chem. Phys. 140, 121105 (2014).

[29] H. Li, Y. Guo, and J. Robertson, Phys. Rev. Mat. 2, 074601 (2018); Sci. Rep. 7, 16858 (2017).

[30] S. J. Clark and J. Robertson, Phys. Rev. B 82, 085208 (2010).

[31] J. Heyd, G. E. Scuseria, and M. Ernzerhof, J. Chem. Phys. 118, 8207 (2003).

[32] M. Y. Zhang and H. Jiang, Phys. Rev. B 100, 205123 (2019).

[33] W. Gao, W. Xia, Y. Wu, W. Ren, X. Gao, and P. Zhang, Phys. Rev. B 98, 045108 (2018).

[34] J. J. Hall, Phys. Rev. 161, 756 (1967).

[35] E. P. O’Reilly and J. Robertson, Phys. Rev. B 34, 8684 (1986).
[36] E. Fois, A. Selloni, G. Pastore, Q. M. Zhang, and R. Car, Phys. Rev. B 45, 13378 (1992).

[37] G. A. N. Connell and R. J. Temkin, Phys. Rev. B 9, 5323 (1974).

[38] See Supplemental Material at http://link.aps.org/supplemental/ 10.1103/PhysRevMaterials.4.054603 for the $\mathrm{Cu}-\mathrm{Cu}$ clustering.

[39] S. Hull and D. A. Keen, Europhys. Lett. 23, 129 (1993).

[40] S. Hull, D. A. Keen, W. Hayes, and N. J. G. Gardner, J. Phys. Cond. Matt. 10, 10941 (1998); S. Hull, Rep. Prog. Phys. 67, 1233 (2004).

[41] D. A. Keen, S. Hull, A. C. Barnes, P. Berastegui, W. A. Crichton, P. A. Madden, M. G. Tucker, and M. Wilson, Phys. Rev. B 68, 014117 (2003).

[42] J. Robertson, J. Phys. C 12, 4767 (1979).

[43] J. Robertson, J. Non-Cryst. Solids 358, 2437 (2012).

[44] L. Friedman, J. Non-Cryst. Solids 6, 329 (1971).

[45] T. Kamiya, K. Nomura, and H. Hosono, Appl. Phys. Lett. 96, 122103 (2010); R. Martins, P. Barquniha, A. Pimentel, L. Periera, and E. Forunato, Phys. Status Solidi A 202, R95 (2005); T. Kamiya, K. Nomura, and H. Hosono, J. Display Technol. 5, 273 (2009). 